\title{
Bridge damage detection and quantification under environmental effects by Principal Component Analysis
}

\author{
Fernando J. Tenelema ${ }^{1}$, Rick M. Delgadillo ${ }^{1}$ and Joan R. Casas ${ }^{1}$ \\ ${ }^{1}$ Technical University of Catalonia (UPC-BarcelonaTech), Barcelona 08034, Spain \\ fernando-josue.tenelemalestudiant.upc.edu
}

\begin{abstract}
Monitoring structural damage is widely used for sustaining and preserving the service life in civil structures, especially in bridges. The influence of environmental variability like temperature affects the dynamic behavior, which can mask subtler structural changes caused by damage. The direct application of vibration-based damage detection methods to measured responses without a prior treatment of the ambient data may lead to false condition assessments. In this article, the main objective is to separate the structural damage conditions from the changes caused by the environmental effects in a numerical benchmark bridge. The Principal Component Analysis (PCA) is applied to decide if the change in vibration characteristics is due to environmental effects or structural damages. The proposed approach in the use of PCA not only allows to detect the damage without the requirement of the baseline to consist of damage sensitivity features obtained from a wide range of environmental conditions, but also serves as a measure for its quantification. The effectiveness and robustness of the proposed methodology is applied to a benchmark bridge structure generated as part of COST Action TU1402 on quantifying the value of information (VoI) in SHM. The benchmark model consisted of a two-span steel bridge under environmental effects, in which two levels of damage were introduced.
\end{abstract}

Keywords: Damage identification, Modal frequency, Temperature effect, Principal Component Analysis.

\section{$1 \quad$ Introduction}

Operational and environmental variability (OEV) in bridges is one of the most complex challenges for developing reliable SHM systems. The effects of OEV can often mask subtler structural changes caused by structural damage [1]. In other words, measurement data recorded by sensors demonstrate sensitivity to structural damage as well as to any change in operational and environmental conditions. Therefore, the direct application of vibration-based damage detection methods to measured responses without a prior treatment of the ambient data may lead to false condition assessments. Regarding the presence of environmental variability, humidity and temperature are the main environmental conditions affecting the bridge structural performance. Temperature is of particular interest since it is a global ambient condition, whose variations considerably change the recorded waveform and vibrational characteristics, 
such as frequency, mode shapes, and damping ratio [2-3]. In fact, many investigations stated that temperature is a critical source that can cause a significant variability of modal frequency up to $10 \%$ for highway bridges [4-5]. And, in most cases, these temperature-induced changes in modal frequencies exceed those caused by structural damage [6]. Therefore, in order to remove or filter the environmental effects from damage effects different methods have been proposed.

This paper investigates damage detection in a numerical benchmark developed by $\mathrm{K}$. Tatsis and E. Chatzi [7] through analysis of natural frequency data using the PCAbased temperature filtering method presented by Soo et al. [8]. On the one hand, the numerical model [7] consists of a two-span steel girder bridge and includes six levels of damage simulated in two different locations: one where the damage is located in the center of the left span and the other where damage is over the intermediate elastic support. On the other hand, a modal analysis of the numerical bridge can be performed since natural frequency variations due to ambient excitations are linear, hence the application of PCA is accurate to deal with the extracted data set.

Results demonstrate that the proposed method is efficient and accurate in detecting damage under temperature variations. The reliability of these methods for damage detection encourages their implementation in the SHM of more complex structures.

\section{Damage Detection Method}

\subsection{Principal Component Analysis (PCA)}

PCA plays an important role in SHM, particularly, in dimensional reduction and feature selection. PCA is a well-known linear method for data analysis, which is used for mapping multidimensional data and as a dimensional reduction tool [8]. Besides, damage sensitivity features collected from bridge structures subjected to environmental conditions can be processed by PCA to extract the main factors driving the variances in the data set [7]. Herein, PCA not only reduces the dimensions of the original data set, but also points out the differences and similarities in the original data set.

$\mathrm{Z}$ denotes a $\mathrm{n} \mathrm{x} \mathrm{m}$ data set of damage sensitivity features collected from $\mathrm{m}$ observations with $\mathrm{n}<\mathrm{m}$. In this study, only the natural frequencies are used as damage sensitive parameter which is represented by $n$ and $m$ represents the number of observations or experimental trials taken at different temperature conditions, as follows:

$$
Z=\left[\begin{array}{ccc}
z_{1,1} & \cdots & z_{1, \mathrm{~m}} \\
\vdots & \ddots & \vdots \\
z_{\mathrm{n}, 1} & \cdots & z_{\mathrm{n}, \mathrm{m}}
\end{array}\right]
$$

Before the application of PCA, the original data matrix $\mathrm{Z}$ must be normalized to have zero mean and unity variance resulting in a standardized matrix which is indicated as $X_{n \times m}$. Then, PCA is applied to $X$ which consists of transforming the data set $X$ into a new $\mathrm{p} \times \mathrm{m}$ data set $\mathrm{Y}$ with smaller dimensions which characterizes most of the 
variances in the original data set. In this regard, a transformation matrix $\mathrm{T}$ is used to relate the $\mathrm{Y}$ and $\mathrm{X}$, which has dimensions $\mathrm{p} \mathrm{x} \mathrm{n}$ as shown in Eq. (2).

$$
Y_{p x m}=T_{p x n} X_{n \times m}
$$

$\mathrm{T}$ is called loading matrix and its rows correspond to the eigenvectors of the covariance matrix of $\mathrm{X}$. To compute the eigenvalues and eigenvectors of the covariance matrix, the Singular Value Decomposition (SVD) procedure is applied. Y is the score matrix and it represents a new set of data which combines the scores of each observation obtained for the factors affecting the original data set. Therefore, damage can be detected by considering the analysis of only the first few rows of the score matrix (first few principal components or T-scores in PCA method). For this reason, the number of principal components should be chosen carefully in order to avoid the false alarms [8].

\subsection{Damage detection methodology based on natural frequency}

Based on a simulated truss structure model, Soo et al. [8] selected the first four natural frequencies as damage-sensitive features from two extreme and opposite environmental conditions (baseline) to create the data matrix. Since the data set is obtained from two extreme and opposite temperature conditions of the undamaged structure, Soo et al. stablished that the effects of temperature variations are represented in the PC1. Consequently, the extreme cases are represented on opposite sides in the PC1 graph, showing negative and positive values of the variance as long as the data set is standardized. Therefore, the other principal components will represent other minor factors affecting the data set such as structural damages. The PCA methodology to filter the temperature effects affecting the bridge can be divided into five main steps:

1. To create the baseline matrix whose rows correspond to the 10 extreme cases including five cases at low temperatures $\left[-30^{\circ} \mathrm{C},-29^{\circ} \mathrm{C},-28^{\circ} \mathrm{C},-27^{\circ} \mathrm{C},-26^{\circ} \mathrm{C}\right]$ and five cases at high temperatures $\left[66^{\circ} \mathrm{C}, 67^{\circ} \mathrm{C}, 68^{\circ} \mathrm{C}, 69^{\circ} \mathrm{C}\right.$ and $\left.70^{\circ} \mathrm{C}\right]$; while their columns correspond to the predominant natural frequencies.

2. To obtain a new observation to the baseline to create the matrix $Z$ which then is standardized to zero mean and unit variance, resulting in the matrix $X$.

3. To obtain the T-scores of each principal component once PCA is applied to X

4. To plot the T-scores of every principal component. Then, if the PCs whose Tscores obtained from the baseline have a separation between them, then these PCs are retained for damage detection. Conversely, if T-scores obtained from the baseline are mixed together, then its associated PC is discarded as well as the following components.

5. To analyze the retained PCs. If the T-scores corresponding to the new observation lie inside the T-scores from baseline, no damage has occurred, otherwise, it implies the presence of damage. In general, the PC1 reflects the effects of the environmental conditions, whereas the second PC represent the effects of damage. 


\section{Case study - Numerical Benchmark}

\subsection{Numerical Bridge}

The numerical benchmark represents a plane stress problem of the superstructure of a two-span continuous steel girder bridge. The bridge geometry is shown in Figure 1 with a total length of $20 \mathrm{~m}$, a total height of $0.6 \mathrm{~m}$ and a thickness of $\mathrm{b}=0.1 \mathrm{~m}$. The bridge has linear elastic behavior with Young's modulus E $=215 \mathrm{GPa}$, Poisson's ratio $v=0.3$ and material density $\rho=7850 \mathrm{~kg} / \mathrm{m}^{3}$ at ambient temperature of $\mathrm{T}=20^{\circ} \mathrm{C}$.

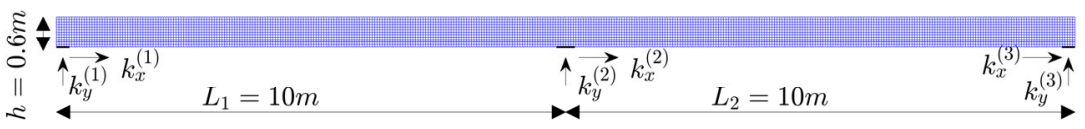

Fig. 1. Flow chart of the PCA-based damage detection method, modified from [9]

The bridge is supported by three equally spaced springs: two springs acting at both ends in a width of $0.3 \mathrm{~m}$ and one intermediate spring acting in the middle of the beam in a width of $0.4 \mathrm{~m}$. All springs are modelled as point supports, each with two degrees of freedom. In order to avoid the mixing of the longitudinal and vertical bending mode shapes, the horizontal and vertical stiffness of springs are $\mathrm{k}_{\mathrm{x}}=10^{6} \mathrm{kN} / \mathrm{m}$ and $\mathrm{k}_{\mathrm{y}}$ $=10^{12} \mathrm{kN} / \mathrm{m}$, respectively. A four-node bilinear isoperimetric element referred as QUAD4 was implemented with full integration by using a 2x2 Gauss quadrature rule. And after a mesh convergence study, a mesh element size of $5 \mathrm{~cm}$-length and $5 \mathrm{~cm}$ height was chosen, resulting in a system of more than 10.000 degrees of freedom.

\subsection{Damage Scenarios}

The structural damage is modelled as a reduction of Young's modulus at the Gauss points on the selected damaged elements. This is commonly known as stiffness reduction (SR). Two damage regions are considered as shown in Figure 2; one where damage is located in the center of the left span $(x=5 \mathrm{~m})$ and the other where damage is over the intermediate elastic support $(x=10 \mathrm{~m})$, covering different numbers of damaged elements and resulting in six damage scenarios (DMG). In order to study the effects of damage severity related to the percentage of SR, the Group of damage 1 (GPD1) is generated. It consists of the undamaged condition of the bridge and the damage scenario 3 (DMG3) with a varying stiffness loss: 50\%, 70\% and 90\%.

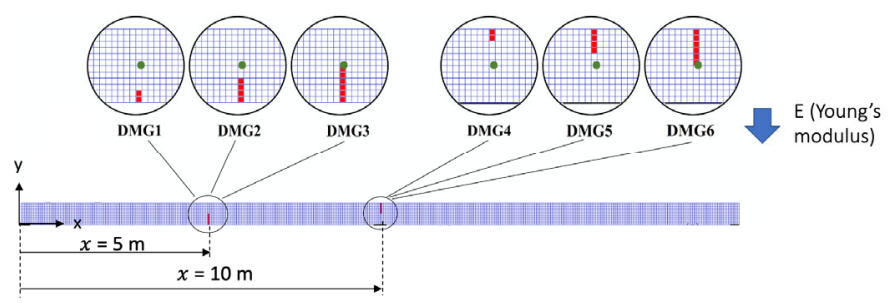

Fig. 2. Damage locations on steel beam (red squares). 


\subsection{Natural Frequency \& Temperature}

A few simplifying assumptions are made regarding the thermo-mechanical behavior of structural steel. On the one hand, Poisson's ratio defined as the ratio of relative contraction to relative expansion of the material is assumed to be unchangeable with temperature. On the other hand, a linear relationship of Young's modulus with temperature is assumed within the ambient temperature of $20^{\circ} \mathrm{C}$ and $100^{\circ} \mathrm{C}$. Young's modulus, E, of steel at these temperatures are obtained based on the laboratory tests of steel S235JR performed by Skowronski et al. [10]. Therefore, $\mathrm{E}=215 \mathrm{GPa}$ at $20^{\circ} \mathrm{C}$, while the Young's modulus at $100^{\circ} \mathrm{C}$ is $\mathrm{E}=202 \mathrm{GPa}$. For simplicity, the intermediate and negative values of Young's modulus are computed as in Eq. (3).

$$
E(T)=218.25-0.1625 T
$$

A modal analysis was firstly performed to obtain the natural frequencies of the bridge and their corresponding mode shapes. The natural frequencies of the bridge considered for this particular benchmark only correspond to the predominant bending modes (y direction) having a cumulative effective mass participation factor (EMPF) between $80 \%$ and $90 \%$. It should be recalled that the EMPF represents the quantity of the system mass participating in a particular mode for a particular direction. Therefore, the larger the EMPF of a particular mode, the greater the contribution of this mode to the dynamic response. For this case study, the modes $2,3,4,5,7$ and 8 are considered having a cumulative EMPF of $82.97 \%$. The selection criteria of predominant modes for this benchmark is explained in more detail in Tenelema [11].

\section{$4 \quad$ Results and discussion}

\subsection{Natural Frequency}

The results of the first two principal components (T-scores) corresponding to three undamaged cases with temperatures of $-10^{\circ} \mathrm{C}, 20^{\circ} \mathrm{C}$ and $40^{\circ} \mathrm{C}$ are illustrated in Figure 3 . The first 10 observations represent the extreme cases, where the first five observations (blue dots) represent the extreme cases at very low temperatures (coldest limit) and the following five observations (red dots) represent the extreme cases at very high temperatures (hottest limit). The eleventh observation (green dot) represents the monitored case. For the undamaged cases, only the PC1 is retained for analysis of damage detection since in the following principal components the baseline observations are mixed together (see figure 3 right). Consequently, for all the undamaged cases under the varying temperature condition, the proposed method does not give rise of damage alerts since the monitored case (green dot) lies inside the baseline. Moreover, it can be observed how the monitored case moves from the coldest limit $\left(\sim-30^{\circ} \mathrm{C}\right)$ to the hottest limit $\left(\sim 70^{\circ} \mathrm{C}\right)$ with increasing temperature (from top to bottom of the figure). Taken the first undamaged monitored case as a general example, the T-scores obtained for the extreme temperatures $-30^{\circ} \mathrm{C}\left(\right.$ Obs. 1 ) and $70^{\circ} \mathrm{C}$ (Obs. 10) are 2.3659 and -2.6454 , respectively, while the $\mathrm{T}$-scores obtained for the monitored case is 1.3787 . Then, the temperature condition for the monitored case can be obtained by linear interpolation 
of these values, resulting in approximately $-10.299^{\circ} \mathrm{C}$, close enough to the actual imposed temperature of $-10^{\circ} \mathrm{C}$. Therefore, the T-scores can be used as a rough indicator to assess the temperature condition of the structure without any direct measurement of the temperature.

Figure 4 illustrates the results for the damaged cases of GPD1 at $20^{\circ} \mathrm{C}$. In this case, the first two PCs are retained since the low-and-high-temperature baseline observations are not mixed with each other. The plots of the PC1 for all damaged cases (left column of the figure) show the monitored case between the two extreme cases, hence the temperature condition of the structure is found within $-30^{\circ} \mathrm{C}$ and $70^{\circ} \mathrm{C}$. However, it can be noticed that as the severity of damage increases, the monitored case begins to move from the coldest limit $\left(\sim-30^{\circ} \mathrm{C}\right)$ to the hottest limit $\left(\sim 70^{\circ} \mathrm{C}\right)$ as if there had been an increase in the temperature of the system when in fact there is not. This can lead to an unreliable estimate of the actual temperature. Tenelema [11] suggested that the reason for this issue lies in the fact that some of the selected modes are very sensitive to damage. For this benchmark, the low-frequency modes (modes 2 and 3) and highfrequency modes (modes 7 and 8) cause notable changes in the vibration features when damage occurs, particularly for high values of SR, whereas the intermediatefrequency modes (modes 4 and 5) have less impact on these features, but still can detect damage. Since all these modes have been firstly considered, when the severity of damage increases, the natural frequencies largely reduce, then a small part of this frequency reduction caused by damage is misunderstood as an increase of the temperature shown as an offset of the monitored case in the first principal component.
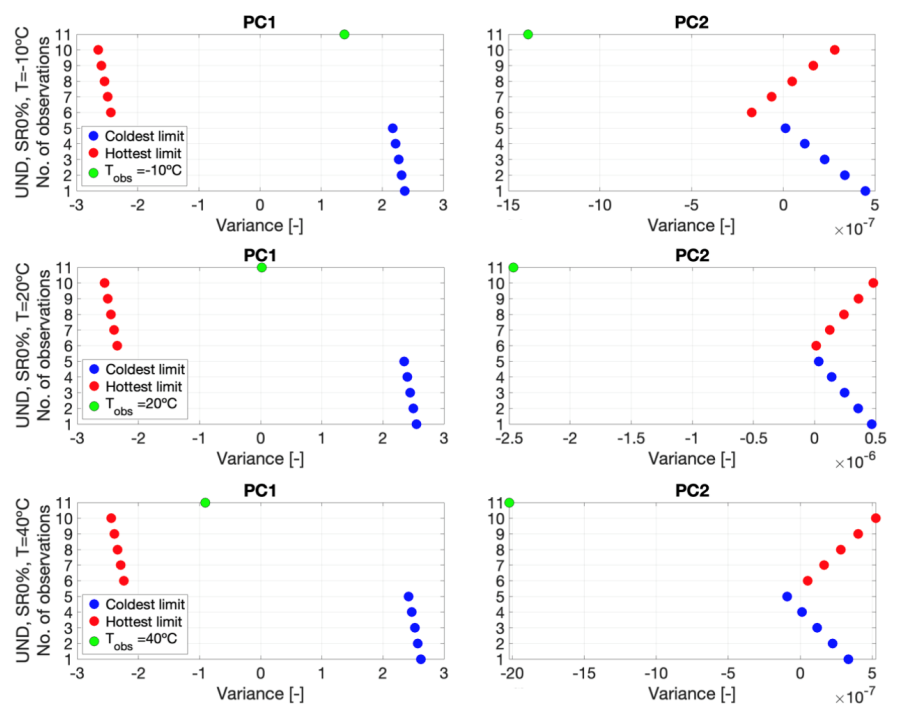

Fig. 3. Graph of first and second principal components for the undamaged cases at $-10^{\circ} \mathrm{C}, 20^{\circ} \mathrm{C}$, and $40^{\circ} \mathrm{C}$ (from top to bottom).

On the other hand, in the case of PC2, an amplification of the results is carried out to have a better analysis and considering only ten cases of the baseline. It can be seen 
from the PC2 that damage alert is raised for all damaged cases since the monitored case (green dot) lies outside the two extreme cases used as baseline having a clear separation between them. This suggests that another factor different from the temperature variation affects the data set, which can be attributed to damage. Damage evolution from case to case can also be seen clearly from the PC2. As the stiffness loss (SR) increases from $50 \%$ to $90 \%$, the difference in the variance (gap) between the low-and-high temperature baseline increases as well, from 0.000144 for SR $50 \%$ to 0.01159 for SR90\%. Moreover, the monitored case has a greater deviation from the baseline when the severity of damage increases. Therefore, the proposed methods are valid not only for detecting damage, but also to quantify it.
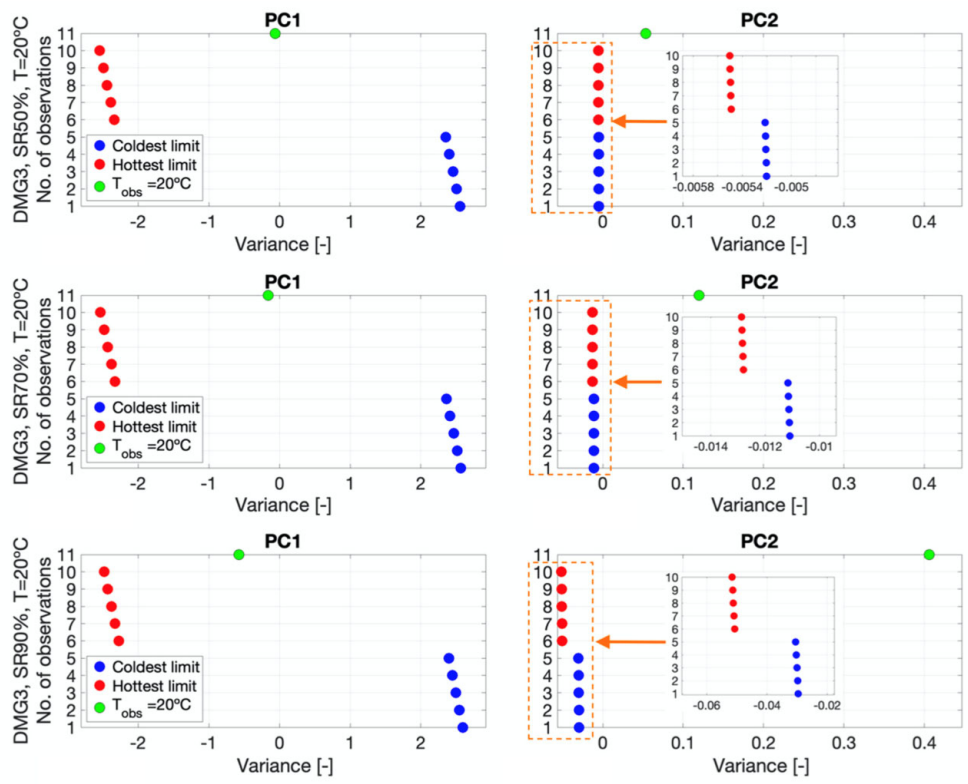

Fig. 4. Graph of first and second principal components for the damage cases corresponding to the GPD1 at $20^{\circ} \mathrm{C}$.

\section{Conclusions}

The results show the effectiveness of the proposed method in distinguishing structural damage from temperature effects regarding the variation in the natural frequencies of the structure. On the one hand, for the undamaged cases subjected to varying temperatures, the $\mathrm{PC} 1$ is the most relevant component accounting for most of the variance in the original data set and having a clear separation between the observations of the baseline. Moreover, the temperature condition of the structure can be assessed without any direct measurement of the temperature. The proposed method shows a good performance in separating the effects of temperature from the structural damage considering the variability in the severity and depth of damage. However, this method also 
presents some drawbacks. For instance, when the level of damage is too large, it is difficult to provide an accurate estimate of the temperature condition. Besides, since natural frequency is a global parameter which is used as a damage sensitive feature, it is not possible to locate the damage. Therefore, in order to locate damage, the use of time-varying parameters obtained from several sensors in the bridge is preferable.

\section{Acknowledgments}

The second author would like to express their gratitude for financial support from Ministry of Education of Peru with the President of the Republic Scholarship for the great support on his PhD studies. All the authors are grateful to Prof. Eleni Chatzi, ETH Zurich, for providing the benchmark bridge data assessed within the present study. Authors are also indebted to the Secretaria d' Universitats i Recerca de la Generalitat de Catalunya for the funding provided through AGAUR (2017 SGR 1481).

\section{References}

1. Y. Liang, D. Li, G. Song, and H. Li, Damage Diagnosis under Environmental and Operational Variations Using Improved Restoring Force Method, in Earth and Space 2014, 2015, pp. 690-702

2. P. Cawley, Long range inspection of structures using low frequency ultrasound, Structural Damage Assessment Using Advanced Signal Processing Procedures, pp. 1-17, 1997.

3. R.M. Delgadillo, J.R. Casas, "Non-modal vibration-based methods for bridge damage identification. Structure and Infrastructure Engineering", 16:4, 676-697.

4. A. Cunha, E. Caetano, F. Magalhães, and C. Moutinho, "Recent perspectives in dynamic testing and monitoring of bridges," Structural Control and Health Monitoring, vol. 20, no. 6, pp. 853-877, 2013.

5. J. Brownjohn, R. Westgate, K. Koo, and E. Cross, "Identifying and discriminating thermal effects for structural health monitoring," in Proceedings of the Second Conference on Smart Monitoring, Assessment and Rehabilitation of Civil Structures (SMAR 2013), 9th11th September, Istanbul, Turkey, 2013.

6. J. Ko and Y. Q. Ni, "Technology developments in structural health monitoring of largescale bridges," Engineering structures, vol. 27, no. 12, pp. 1715-1725, 2005

7. K. Tatsis and E. Chatzi, A numerical benchmark for system identification under operational and environmental variability, in Proceedings of the 8th International Operational Modal Analysis Conference (IOMAC 2019), Copenhagen, Denmark, 2019.

8. W. Soo Lon Wah, Y.-T. Chen, G. W. Roberts and A. Elamin, Separating damage from environmental effects affecting civil structures for near real-time damage detection, Structural Health Monitoring, vol. 17, no. 4, pp. 850-868, 2018.

9. U. Demšar, P. Harris, C. Brunsdon, A. S. Fotheringham, and S. McLoone, "Principal component analysis on spatial data: an overview," Annals of the Association of American Geographers, vol. 103, no. 1, pp. 106-128, 2013.

10. W. Skowroński, A. Włóka, and R. Chmiel, "Modelling of strength processes of S235JR steel at increased temperature," Structure and Environment, vol. 6, 2014.

11. F. J. Tenelema, Bridge Damage Identification under operational and environmental variability, Master's thesis, Barcelona, Spain, 2020. 